\title{
Prevalence of methicillin-resistant Staphylococcus aureus (MRSA) in nasal swab specimens obtained from hospitalized patients and healthcare workers in a Belgrade hospital
}

\author{
B Jovanovic ${ }^{*}$ Ivana Cirkovic \\ From International Conference on Prevention \& Infection Control (ICPIC 2011) \\ Geneva, Switzerland. 29 June - 2 July 2011
}

\section{Introduction / objectives}

The aim of the present study was to provide the analysis of carriage of MRSA in hospitalized patients and HCWs in the largest healthcare facility in Serbia.

\section{Methods}

Nasal swab were taken from 195 hospitalized and 105 HCWs at the Clinical Center of Serbia in Belgrade. Each swab was inoculated directly onto MRSA-ID agar (bioMérieux, France). All inoculated solid media were incubated at $35^{\circ} \mathrm{C}$ and read after $24 \mathrm{~h}$ and $48 \mathrm{~h}$ of incubation. Identification of isolates was confirmed by PCR for $n u c$ and mecA gene. Susceptibility to antibiotics was performed by disk diffusion method in accordance to the CLSI recommendations. Determination of SCCmec types was done by previously described PCR protocol.

\section{Results}

Among 195 hospitalized patients and 105 HCWs, 23 $(11.8 \%)$ and 8 (7.6\%) respectively were colonized MRSA. All tested MRSA strains were susceptible to fusidic acid, trimethoprim/sulfamethoxazole, vancomycin, linezolid, pristinamycin and mupirocin, while $27(87.1 \%)$ were resistant to gentamicin, $28(90.3 \%)$ to kanamycin, 27 (87.1\%) to tobramycin, 17 (54.8\%) to erythromycin, 17 (54.8\%) to clindamycin, 25 (80.6\%) to ciprofloxacin, 3 $(9.7 \%)$ to rifampin, $4(12.9 \%)$ to tetracycline and 5 (16.1\%) to chloramphenicol. Among MRSA strains isolated in this study, 6 (19.4\%) strains could be classified as CA-MRSA, because they were SCCmec type IV or V and most of them were susceptible to all tested antibiotics except beta-lactams. The remaining 25 (80.6\%) MRSA strains had characteristics of HA-MRSA, they were SCCmec I or III, and all were resistant to different antibiotics beside beta-lactams.

\section{Conclusion}

Carriage of MRSA among hospitalized patients and HCWs was determined to be high, $10.3 \%$. Carriage was higher in hospitalized patients than in HCWs. Most of the isolated strains were HA-MRSA.

\section{Disclosure of interest}

None declared.

Published: 29 June 2011

\section{doi:10.1186/1753-6561-5-S6-P16}

Cite this article as: Jovanovic and Cirkovic: Prevalence of methicillin-

resistant Staphylococcus aureus (MRSA) in nasal swab specimens obtained from hospitalized patients and healthcare workers in a Belgrade hospital. BMC Proceedings 2011 5(Suppl 6):P16.

Hospital Epidemiology, Clinical Center of Serbia, Belgrade, Serbia 